\title{
Preparation of Nanopatterned Polyimide by Imprinting and Curing Phenylethynyl- terminated Imide Oligomer
}

\author{
Masahiko Ogino ${ }^{1}$, Tatsuya Ohashi $^{1}$, Hirofumi Yoshida ${ }^{1}$, \\ Daigo Nagayama ${ }^{2}$, Toshio Kubota ${ }^{2}$, Atsushi Morikawa ${ }^{2}$ and Katsumichi Ono ${ }^{2}$ \\ ${ }^{1}$ Hitachi Research Laboratory, Hitachi, Ltd., Hitachi, Ibaraki 319-1292, Japan \\ ${ }^{2}$ Department of Biomolecular Functional Engineering, Ibaraki University, Hitachi, \\ Ibaraki 316-8511, Japan
}

\begin{abstract}
Addition-type phenylethynyl-terminated imide oligomers were synthesized from 4,4'-diaminodiphenyl ether(ODA), 4-phenylethynylphthalic anhydride (PEPA) and 3,3',4,4'-biphenyltetracarboxylic dianhydride (BPDA) or 4,4'-hexafluoroisopropylidene)dianhydrid (6FDA), and preparation of nanopatterned polyimide was examined by imprinting the melted phenylethynyl-terminated imide oligomer and crosslinking the ethynyl groups. Crosslinked polyimide films were prepared by pressing the melted phenylethynyl-terminated imide oligomers and curing them under pressure at $370{ }^{\circ} \mathrm{C}$ afterward, and the properties of the polyimide films were studied for comparison. The crosslnked polyimide films had high glass transition temperature $(\mathrm{Tg})$ above $320{ }^{\circ} \mathrm{C}$, and showed excellent tensile strength more than $100 \mathrm{MPa}$. Nanopatterned polyimide was prepared by imprinting the melted imide oligomer with a metal mold and curing it under pressure at $370{ }^{\circ} \mathrm{C}$ afterward. ZEONOR ${ }^{\circledR}$ film could be imprinted using the imprinted and cured imide oligomer as sub-mold instead of the metal mold.
\end{abstract}

Keyword: imprint, phenylethynyl-terminated imide oligomer, crosslink, nanopatterned polyimide

\section{Introduction}

In the nanoimprint lithography (NIL), a mold with nanopattern on its surface is pressed into a thin resist cast on a substrate [1-4]. The resist, a thermal plastic, is deformed readily by the mold when heated above $T \mathrm{~g}$ due to low viscosity. After the resist is cooled below $T \mathrm{~g}$, the mold is removed. The surface of the resist is changed into the shape opposite to the mold pattern. We investigated to form nanopattern on polyimide surface by NIL using metal mold and application of the nanopatterned polyimide to sub-mold for formation of the nanopattern on other polymer surface.

Aromatic polyimides are well known to have high dimensional stability, low thermal expansion, outstanding thermal and mechanical properties, as well as environmental stability [5]. Therefore, nanopattern formed on polyimide is expected to be tough and durable. Recently, the nanopatterns have been fabricated on polyimide films to use in various fields, such as microelectric devices [6], biological applications [7] and displays [8], and their application could be further extended to other fields if they can be easily patterned at micro or nanoscale. Aromatic polyimide, Pyralin 2525 (HD Microsystems PI2525), has been imprinting by three approaches; imprint as its uncured soft state and cure it afterwards; imprint another low $T \mathrm{~g}$ polymer, then form the pattern on polyimide surface by oxygen reactive ion etching; and direct imprint on polyimide surface at temperature higher than its $T \mathrm{~g}$ [9]. Since aromatic polyimides do not exhibit high molecular mobility even at 
temperatures higher than their $\mathrm{Tg}$, the processing conditions for molding are extremely severe, or polyimide films are prepared by conversion of soluble polyamic acids, the precursors of polyimide [5]. Nanoimprinted Kapton-typepolyimide has already been obtained by imprinting the polyamic acid film and thermal imidation afterward [10]. Though a shrinkage took place during thermal imidation, it did not damage the nanopattern. The optimized nanoimprinting process was proposed by investigating the thermal and dynamic mechanical properties of the polyamic acid and polyimide carefully.

In this study, preparation of nanopatterned polyimide was examined by imprimting addition-type phenylethynyl-terminated imide oligomer. Fluid melted imide oligomer was imprinted and cured under pressure to crosslink the ethynyl groups. The imprinted and cured imide oligomer was evaluated as sub-mold for imprinting ZEONOR ${ }^{\circledR}$ film.

\section{Experimental}

\subsection{Materials}

3,3',4,4'-biphenyltetracarboxylic dianhydride (BPDA), 4,4'-hexafluoroisopropylidene)dianhydrid (6FDA) and 4,4'-diaminodiphenyl ether(ODA) were purchased from Wako Pure Chemical Industry and purified by sublimation under reduced pressure. 4-Phenylethynyl- phthalic anhydride (PEPA) was kindly supplied from Manac Incorporated. $N$-Methyl-2-pyrrolidon (NMP, dehydrated grade) was purchased from Kanto Kagaku and used as received. Upilex $\mathrm{R}$ was purchased from Ube Industries LTD. ZEONOR ${ }^{\circledR}$ was purchased from Zeon Corporation.

\subsection{Synthesis of the imide oligomers (BPDA- PEPA -2)}

In a three necked flask, $0.588 \mathrm{~g}(2 \mathrm{mmol})$ of BPDA was added to a solution of $0.600 \mathrm{~g}(3 \mathrm{mmol})$ of ODA in $20 \mathrm{~mL}$ of NMP, and stirred at room temperature for $5 \mathrm{~h}$. $0.993 \mathrm{~g}(2 \mathrm{mmol})$ of PEPA was added to the homogeneous solution and stirred. After PEPA was dissolved, the reaction solution was stirred for $5 \mathrm{~h}$. $0.612 \mathrm{~g}(6 \mathrm{mmol})$ of acetic dianhydride and $0.470 \mathrm{~g}(6 \mathrm{mmol})$ of pyridine were added to the solution in this order, and stirred for 5 h. The solution was poured into $800 \mathrm{~mL}$ of methanol. The precipitated polyimide oligomer was filtrated, washed with methanol and dried under vacuum.
2.3. Preparation of cured polyimide oligomer film

About $0.3 \mathrm{~g}$ of the imide oligomer powder (BPDA-PEPA-m) was spread in film frame $(80$ $\mathrm{mm} \times 50 \mathrm{~mm} \times 50 \mu \mathrm{m}$ ), and pressed under $2 \mathrm{MPa}$ at $370{ }^{\circ} \mathrm{C}$ for $60 \mathrm{~min}$. Cured film (6FDA-PEPA-m) was prepared under $5 \mathrm{MPa}$. The cured oligomers were obtained as tough films.

\subsection{Procedure of preparing nanopatterned cured imide oligomer}

Six kinds of Si-molds having holes pattern with $1200 \mathrm{~nm}$ depth and different diameters were used for forming pillars patterns on polyimide surface. The diameters of holes were $1,000 \mathrm{~nm}, 500 \mathrm{~nm}$, $350 \mathrm{~nm}, 250 \mathrm{~nm}, 200 \mathrm{~nm}$ and $160 \mathrm{~nm}$.

The Si-mold was treated with release agent as following. The mold was soaked in $0.1 \mathrm{wt} \%$ solution of Optool DSX (Daikin Industries, Osaka, Japan) in Demnumsolvent (Daikin Industries, Osaka, Japan) for 1 minute. After the Si-mold was baked at $120{ }^{\circ} \mathrm{C}$ for $5 \mathrm{~min}$, it was washed with Demnumsolvent.

Imprinting and curing were carried out using 40TON vacuum press machine (Kitagawa seiki, Hiroshima, Japan). The powder of BPDA-PEPA-2 was placed on silicon wafer, and heated. When the powder melted, the Si-mold was contacted and pressed. The temperature was ramped to $370{ }^{\circ} \mathrm{C}$, and held at $370{ }^{\circ} \mathrm{C}$ for $1 \mathrm{~h}$. The imide oligomer was held under $2 \mathrm{MPa}$ between the Si-mold and the silicon wafer during the process. After cooling to room temperature, the patterned cured imide oligomer was removed from the Si-mold. Holes pattern opposite to that of the Si-mold was formed on the surface of the cured imide oligomer.

\subsection{Procedure of imprinting ZEONOR ${ }^{\circledR}$}

Ni-mold having pillars pattern with $500 \mathrm{~nm}$ diameter and $1,000 \mathrm{~nm}$ height was used for preparing holes patterned cured imide oligomer. The Ni-mold was treated with the release agent as the Si-mold. Imprinting and curing were carried out using 40TON vacuum press machine. The powder of 6FDA-PEPA-3 was placed on silicon wafer, and heated. When the powder melted, the $\mathrm{Ni}$ - mold was contacted and pressed. The temperature was ramped to $370{ }^{\circ} \mathrm{C}$, and held at $370{ }^{\circ} \mathrm{C}$ for $60 \mathrm{~min}$. The imide oligomer was held under $5 \mathrm{MPa}$ between the Ni-mold and silicon wafer during the process. After cooling to room temperature, the imprinted and cured imide oligomer was removed from the Ni- mold. The 
cured 6FDA-PEPA film having holes pattern was prepared.

ZEONOR ${ }^{\circledR}$ film was placed on silicon wafer, and heated. The film was imprinted at $200{ }^{\circ} \mathrm{C}$ by the imprinted and cured 6FDA-PEPA, and held under $0.5 \mathrm{MPa}$ at this temperature for $1 \mathrm{~min}$. After cooling to room temperature, ZEONOR ${ }^{\circledR}$ film was removed from the imide oligomer mold. The pillars pattern was formed on the surface of ZEONOR ${ }^{\circledR}$ film.

\subsection{Measurement}

Differential scanning calorimetry (DSC) was performed using a Shimadzu DSC-60 (Shimadzu Corporation, Kyoto, Japan) at a heating rate of $10{ }^{\circ} \mathrm{C} \mathrm{min}-1$. Dynamic viscosity of polyimide oligomer and dynamic mechanical behavior of cured polyimide oligomer were performed with an Advanced Rheometric Expansion System (TA Instruments - Waters LLC, New Castle, Delaware, USA), and the measurements were carried out at $1 \mathrm{~Hz}$ at $5{ }^{\circ} \mathrm{C} \mathrm{min}^{-1}$. Dynamic viscosity and dynamic mechanical behavior were measured using parallel plate and film plate, respectively. The tensile tests of cured films were performed at a tensile speed of $10 \mathrm{~mm} \mathrm{m^{-1 }}$ on specimens of $30 \mathrm{~mm} \times 5 \mathrm{~mm} \times 50 \mu \mathrm{m}$ using Shimadzu AGS-100G (Shimadzu Corporation, Kyoto, Japan). Scanning electron microscopy (SEM) studies were performed using Hitachi S-2300 (Tokyo, Japan).

\section{Results and Discussion}

\subsection{Direct imprint to Upilex-R polyimide}

First, Upilex-R polyimide $\left(\mathrm{Tg}=300{ }^{\circ} \mathrm{C}\right)$ film was examined to be imprinted directly. The nanopatterning PMMA $\left(\mathrm{Tg}=110{ }^{\circ} \mathrm{C}\right)$ had been successfully carried out by imprinting at $175{ }^{\circ} \mathrm{C}$ under $4.4 \mathrm{MPa}$ [1]. The polyimide film was imprinted by a metal mold with holes pattern at $370{ }^{\circ} \mathrm{C}$ under $6 \mathrm{M} \mathrm{Pa}$ for $60 \mathrm{~min}$. Figure 1 shows SEM photography of topography image on the surface. Straightforward pillars patterns could not be obtained on Upilex-R polyimide surface in spite of the severe conditions, and the heights of the pillars were much shorter than the depths of the holes of the mold. The mold patterns could not be imprinted sufficiently due to the low mobility. Upilex-R polyimide shows high modulus of $10^{7} \mathrm{~Pa}$ even at $370{ }^{\circ} \mathrm{C}$, which is much higher than the $\mathrm{Tg}$ [11]. (a)

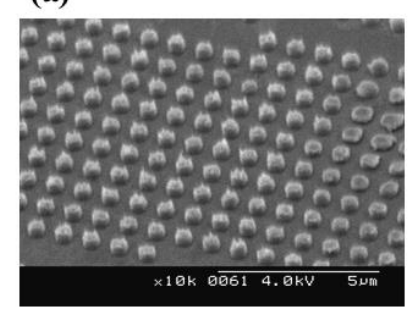

(b)

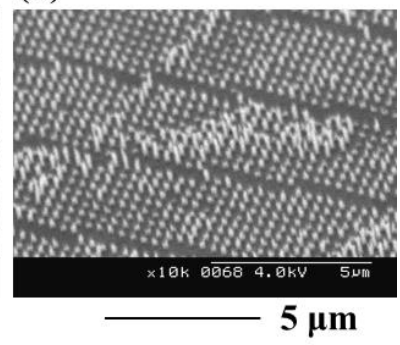

Figure 1. SEM photograph of pillars pattern formed by imprinting Upilex R directly with holles-patterned mold. Holles diameter; (a): $500 \mathrm{~nm}$, and (b): $160 \mathrm{~nm}$.

3.2. Preparation and properties of phenylethynyl-terminated imide oligomer

Sufficient mobility is required to form a pattern, which reflects sufficiently the mold pattern, by imprinting polyimide directly. We examined to imprint processable thermosetting-resin by a metal mold and crosslinking it afterward. Phenylethynyl-terminated imide oligomer ${ }^{12-16}$ was selected as such a resin. It has been examined to fabricate carbon fiber-reinforved plastic from various phenylethynyl-terminated imide origomers [17]. Phenylethynyl-terminated imide origomers were synthesized from the reaction of dianhydrides, ODA and PEPA as shown in Scheme 1. BPDA and 6FDA were used as dianhydride, and imide oligomers having various average degrees of polymerization $(\mathrm{m})$ were synthesized by adjusting the molar ratio. Polyaddition to form polyamic acid, modification of phenylethynyl groups, and chemical solution imidation were carried out through one-pot procedure. The reaction solution was poured into methanol, and the imide oligomer was precipitated as powder. The thermal behaviors of imide oligomers were evaluated by DSC. The imide oligomers showed melting temperature $(\mathrm{Tm})$ at $140-235{ }^{\circ} \mathrm{C}$ and the $\mathrm{Tm}$ values were higher as $\mathrm{m}$ increased. 


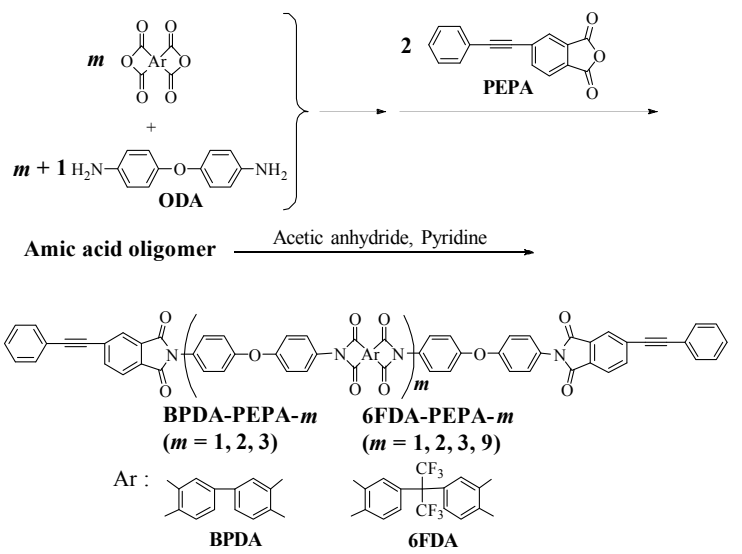

Scheme 1. Synthesis of addition-type phenylethynyl-terminated imide oligomers.

Figure 2 shows temperature dependence the melt viscosities for the imide oligomers, BPDA-PEPA- $\boldsymbol{m}$ and 6FDA-PEPA- $\boldsymbol{m}$. The imide oligomers $(\mathrm{m} \leqq 3)$ had very low melt viscosities $(10 \mathrm{~Pa} \mathrm{~s})$ at $340{ }^{\circ} \mathrm{C}$, and showed increase of the viscosities at about $360{ }^{\circ} \mathrm{C}$ due to crosslinking of the ethynyl groups.

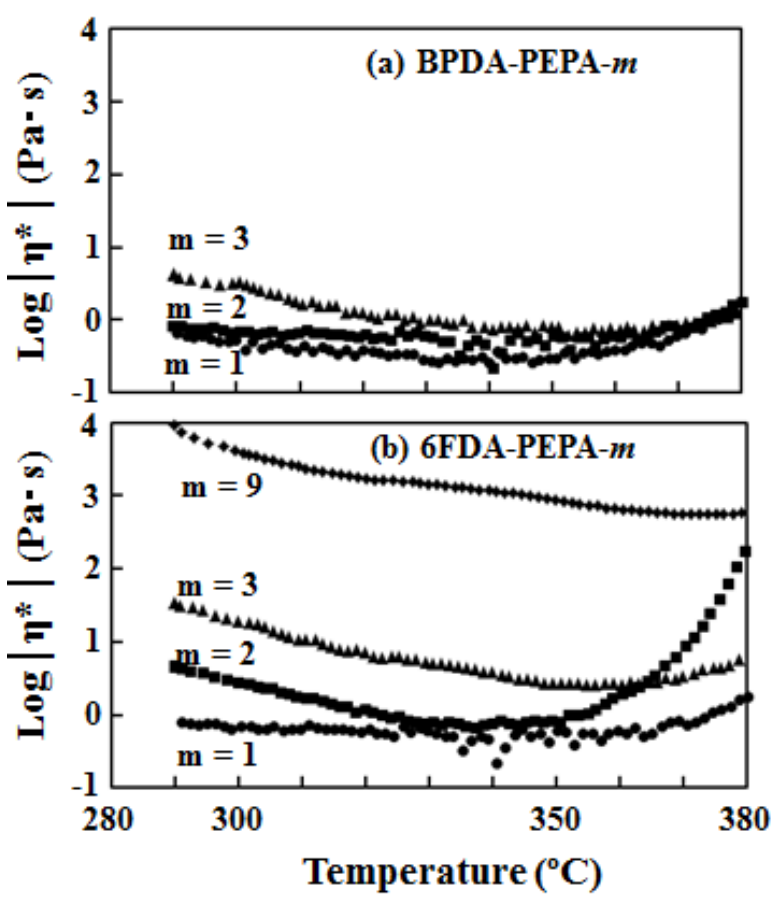

Figure 2. Melting and curing behaviors of the oligomers: (a) BPDA-PEPA- $\boldsymbol{m}$ and (b) 6FDA-PEPA- $m$.
The properties of imide oligomers cured above $360{ }^{\circ} \mathrm{C}$ were examined The powder of imide oligomer was placed in film frame, and held under pressure at $370{ }^{\circ} \mathrm{C}$ for $60 \mathrm{~min}$. The cured oligomers were obtained as tough films. Figure 3 shows dynamic mechanical behaviors of the cured BPDA-PEPA- $\boldsymbol{m}$ and 6FDA-PEPA- $\boldsymbol{m}$. For comparison, those of the corresponding polyimides without phenylethynyl groups, Upilex $\mathrm{R}$ and 6FDA-ODA are also shown. $T g$ values were observed as $\tan \delta$ peak temperatures (Table 1), and $\mathrm{Tg}$ values of the cured imide oligomers were higher than those of the polyimide without ethynyl groups. As $m$ was lower, the $T \mathrm{~g}$ values were higher, the decrements in storage modulus at the glass transition were lower, and the effect of crosslinking was observed in the dynamic mechanical behaviors of the imide oligomers.

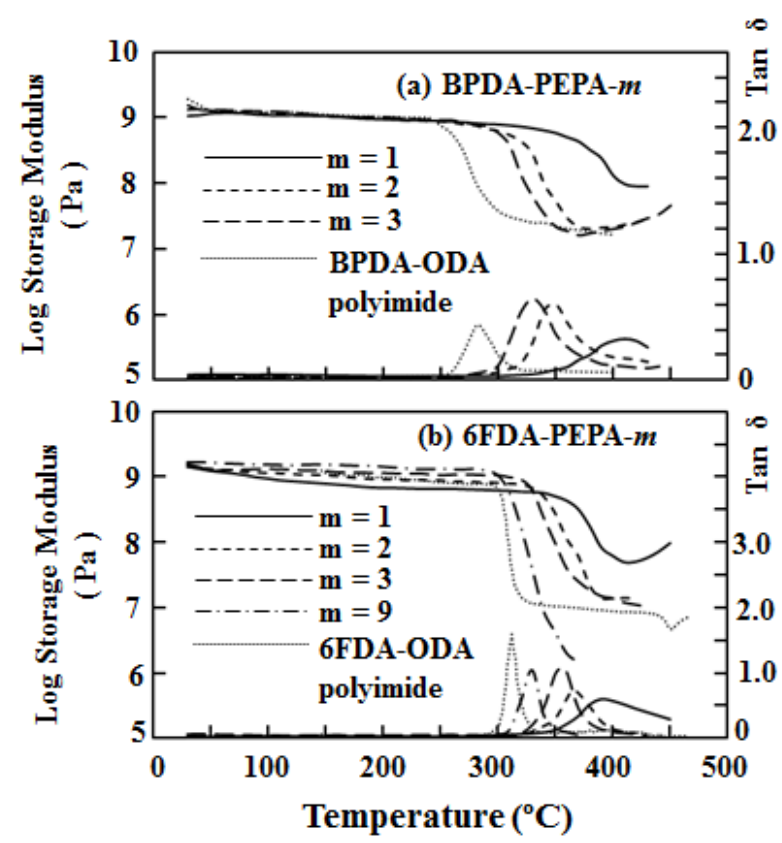

Figure 3. DMA behaviors of the cured oligomers: (a) BPDA-PEPA- $m$ and (b) 6FDA-PEPA- $m$.

The mechanical properties of the cured imide oligomer films were evaluated using tensile tests. Table 1 shows the tensile strength (S), the elongation at break (E) and the tensile modulus (M). All the films had tensile strengths more than $100 \mathrm{MPa}$, and the elongation-at-break was longer with $m$. 
Table 1. Properties of cured imide oligomer films.

\begin{tabular}{lllll}
\hline $\begin{array}{l}\text { Cured imide } \\
\text { oligomer }\end{array}$ & $\begin{array}{l}\mathrm{Tg}^{\mathrm{a}} \\
\left({ }^{\circ} \mathrm{C}\right)\end{array}$ & $\begin{array}{l}\mathrm{S} \\
(\mathrm{MPa})\end{array}$ & $\begin{array}{l}\mathrm{E} \\
(\%)\end{array}$ & $\begin{array}{l}\mathrm{M} \\
(\mathrm{GPa})\end{array}$ \\
\hline BPDA-PEPA-1 & 415 & 119 & 10 & 2.4 \\
BPDA-PEPA-2 & 345 & 127 & 11 & 2.5 \\
BPDA-PEPA-3 & 331 & 128 & 13 & 2.6 \\
6FDA-PEPA-1 & 396 & 124 & 9 & 2.3 \\
6FDA-PEPA-2 & 364 & 129 & 11 & 2.7 \\
6FDA-PEPA-3 & 356 & 140 & 12 & 2.9 \\
6FDA-PEPA-4 & 327 & 148 & 13 & 3.2 \\
\hline
\end{tabular}

${ }^{a}$ Determined by DMA at $5{ }^{\circ} \mathrm{C} \mathrm{min}^{-1}$.

3.3. Preparation of nanopatterned polyimide by imprinting and curing phenylethynyl-terminated imide oligomer

Since the cured imide oligomer films showed high $T \mathrm{~g}$ values and excellent mechanical properties, nanopatterned polyimide was prepared by the similar method. The procedure is shown in Figure 4. The powder of imide oligomer was placed on silicon wafer, and heated. When the powder melted, Si-mold was contacted and imprinted. The temperature was ramped to $370{ }^{\circ} \mathrm{C}$, and held at this temperature for $1 \mathrm{~h}$ to cure the imide oligomer. The imide oligomer was held under pressure between the Si-mold and silicon wafer during the process. BPDA-PEPA-2 and 6FDA-PEPA-3 were imprinted under $2 \mathrm{MPa}$ and 5 $\mathrm{MPa}$, respectively.

Figure 5 shows SEM photography of pillars patterns on the imprinted BPDA-ODA-2 and 6FDA-ODA-3. The diameters and heights of the pillars on the cured polyimide oligomer were agreement with diameters and depths of the holes on the Si-mold surface, and distortion of the pattern was not observed. It was suggested that the melted imide oligomer flowed into all the corners of the holes in the Si-mold and crosslinked without shrinkage.

3.4. Imprint to ZEONOR ${ }^{\circledR}$ film using nanopatterned cured imide oligomer as sub-mold

The patterned cured imide oligomer was evaluated as sub-mold for imprinting ZEONOR ${ }^{\circledR}$ film instead of the metal mold (Figure 7). The melted 6FDA-PEPA-3 was imprinted by Ni-mold with pillars pattern $($ diameter $=500 \mathrm{~nm})$, and cured

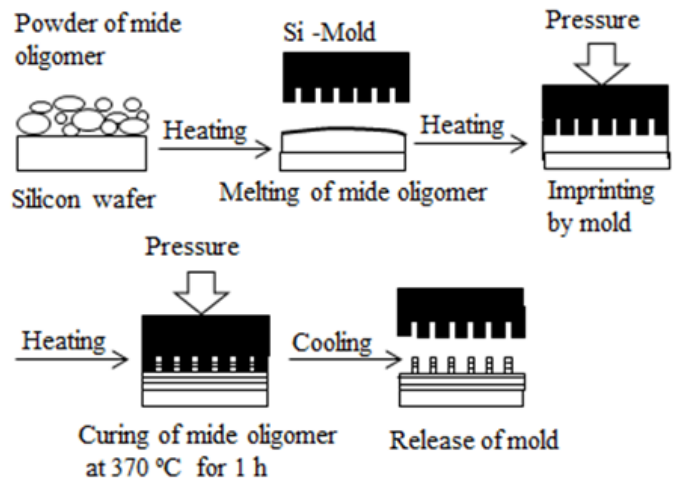

Figure 4. Procedure for preparation of pillars-patterned cured imide oligomer. (a) BPDA-PEPA-2

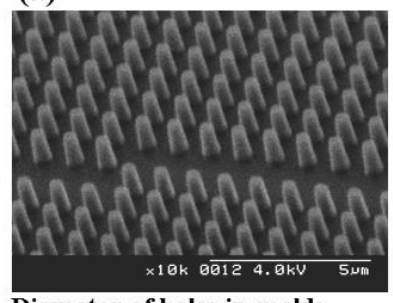

Diameter of holes in mold:

$500 \mathrm{~nm}$

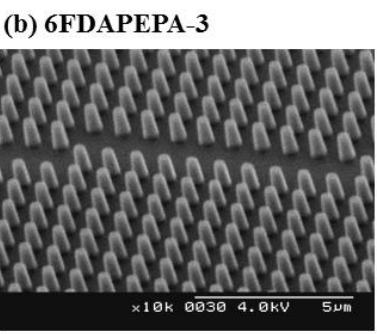

Diameter of holes in mold: $500 \mathrm{~nm}$

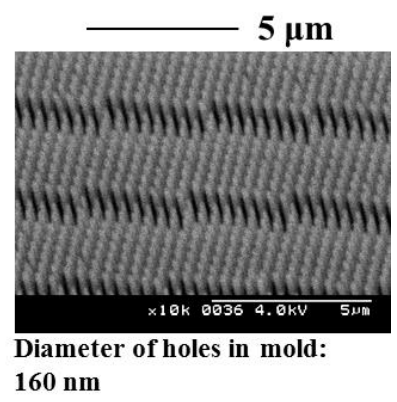

$5 \mu \mathrm{m}$

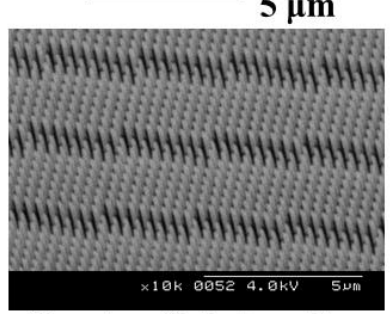

Diameter of holes in mold: $160 \mathrm{~nm}$
Figure 5. SEM photograph of pillars patterns: (a) BPDA-PEPA-2 and (b) 6FDA-PEPA-3.

at $370{ }^{\circ} \mathrm{C}$ for $1 \mathrm{~h}$ afterward (a). ZEONOR ${ }^{\circledR}(T \mathrm{~g}$ $=150{ }^{\circ} \mathrm{C}$ ) was imprinted by the cured 6FDA-PEPA-3, and held under $0.5 \mathrm{MPa}$ at this temperature for $1 \mathrm{~min}(\mathrm{~b})$. Halls pattern, whcih was opposite to the $\mathrm{Ni}$ mold, was formed on the surface of the imprinted and cured 6FDA-PEPA-3, and the same pillars pattern as the Ni-mold was formed on the surface of ZEONOR ${ }^{\circledR}$. 
(a) Preparation of holes-patterned cured 6FDA-PEPA-3

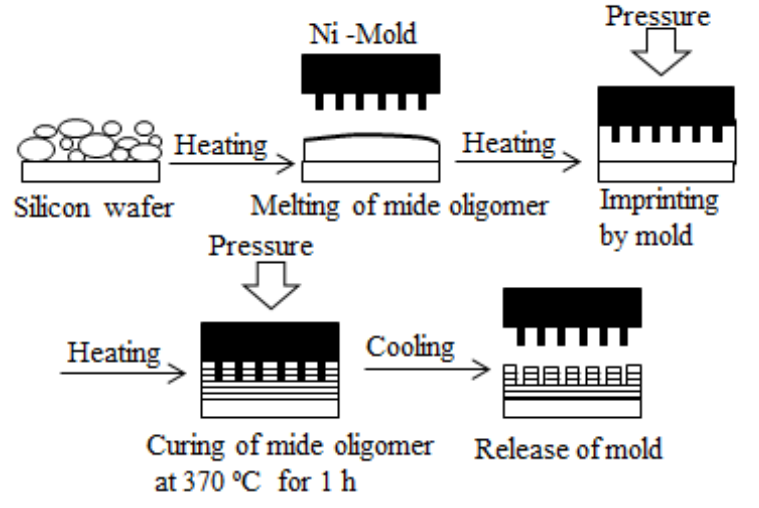

(b) Imprinting ZEONOR ${ }^{\circledR}$ by the cured 6FDA-PEPA-3

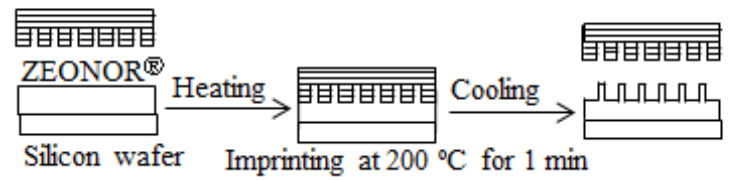

Figure 6. Procudure for imprinting ZEONOR ${ }^{\circledR}$ by using patterned cured 6FDA-PEPA-3 as mold.

Figure 7 shows SEM photography of patterns on the imprinted and cured 6FDA-PEPA-3 and that of the imprinted ZEONOR ${ }^{\circledR}$ film. Though the height of the pillars was slightly longer $(1720 \mathrm{~nm})$, the diameter of pillar was agreement with that of the pillars on the Ni-mold surface, distortion of the pattern was not observed, and the Ni-mold pattern was be reflected sufficiently. The longer pillars is thought to be due to extension occurred in removing the imprinted ZEONOR ${ }^{\circledR}$ film from the mold.

(a)

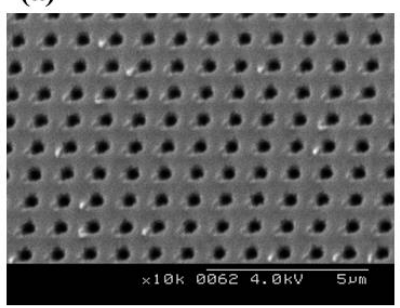

(b)

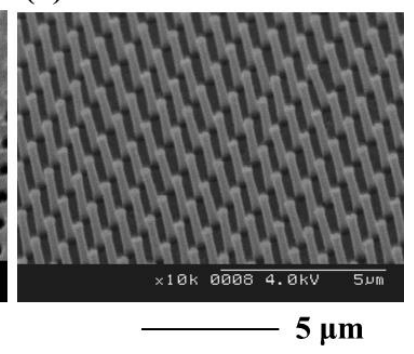

Figure 7. SEM photograph of patterns: (a) patterned cured 6FDA-PEPA-3 and (b) imprinted ZEONOR ${ }^{\circledR}$.

\section{Conclusion}

Addition-type phenylethynyl-terminated imide oligomers with a BPDA-ODA and 6FDA-ODA backbone structures were found to be have good processability for imprinting and conversion property to tough materials by curing them under pressure at $370{ }^{\circ} \mathrm{C}$. Nano-patterns could be formed on the surface by imprinting the melted imide oligomer and cured them under pressure at $370{ }^{\circ} \mathrm{C}$ afterward. The imprinted and cured imide oligomer could be used as sub-mold instead of metal mold for imprinting ZEONOR ${ }^{\circledR}$.

\section{Acknowledgements}

The authors thank Dr Yuich Ishida of JAXA for helpful technical advice.

\section{References}

1. S. Y. Chou, P. R. Krauss and P. J. Rentstrom, J. Vac. Sci. Technol. B 14 (1996), 4129.

2. S. Y. Chou, P. R. Krauss and P. J. Rentstrom, Appl. Phys. Lett. 67 (1995), 3114.

3. S. Y. Chou, P. R. Krauss, W. Zhang, L. Guo and L. Zhuang, J. Vac. Sci. Technol. B 15 (1997), 2897.

4. M. Ogino, M. Hasegawa, K. Sakaue, S. Nagai and A. Miyauchi, Jpn. J. Appl. Phys 52 (2013) 035201 (4 pages) .

5. K. M. Mittal and M. K. Ghosh, in polyimide: Functional and applications (1996) 629 (Marcel Dekker, New York).

6. M. Ree, Macromol. Res. 14 (2006), 1.

7. J. Vinenti, D. -H. Kim, L. Vigerand, E. S. Frechette, J. A. Bianco, Y. S. Kim, A. E. Avrin, V. R. Tiruvadi, S. W. Hwang, A. C. Vanieer, D. F. Wuisin, K. Davis, C. E. Geiber, L. Paimer, J. Van der Spiegel, J. Wu, J. Xiao, Y. Huang, D. Contrenas, J. A. Rogers and B. Litt, Nat. Neurosci. 7 (2008), 277.

8. R. S. Lin and J. A. Roger, Nano. Lett. 7 (2007) 1613.

9. B. Cui, Y. Cortot and T. Veres, Microelectroic Engineering 83 (2006) 906. S.

10. Siqing, H. Wu and A. Takahara, Polym. J. 44 (2012) 1036.

11. C. Chen, R. Yokota, M. Hasegawa, M. Kochi, K. Horie and P. M. Hergenrother, High Perform. Polym. 17 (2005), 313.

12. P. M. Hergenrother and J. G. Smith Jr, Polymer 35 (1994), 4857.

13. P. M. Hergenrother, J. W. Connell and J. G. Smith Jr, Polymer 41 (2000), 5073.

14. J. G. Smith Jr, J. W. Connell and P. M. Hergenrother, J. Comp. Matls. 34 (2000), 614.

15. T. V. Holland, T. E. Glass and J. E. McGrath, Polymer 41 (2000) 4,965.

16. G. W. Meyer, B. Tan, and J. E. McGrath, High Perform. Polym. 6 (1994) 423.

17. M. Miyauchi, Y. Ishida, T. Ogasawara and R. Yokota, Polym. J. 45 (2013) 594. 